\title{
Analyst
}

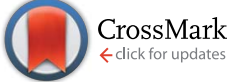

Cite this: DOI: 10.1039/c4an01568a
Received 26th August 2014 Accepted 29th September 2014

DOI: $10.1039 / c 4 a n 01568 a$

www.rsc.org/analyst

\section{Characterization and usage of the EASY-spray technology as part of an online 2D SCX-RP ultra- high pressure system $\uparrow$}

\author{
Fabio Marino, ${ }^{\text {ab }}$ Alba Cristobal, ${ }^{\text {ab }}$ Nadine A. Binai, ${ }^{\text {ab }}$ Nicolai Bache, ${ }^{c}$ Albert J. R. Heck ${ }^{\text {ab }}$ \\ and Shabaz Mohammed*abde
}

Ultra-high pressure liquid chromatography (UHPLC) systems combined with state-of-the-art mass spectrometers have pushed the limit of deep proteome sequencing to new heights making it possible to identify thousands of proteins in a single LC-MS experiment within a few hours. The recently released EASY-spray technology allows one to implement nano-UHPLC with straightforwardness. In this work we initially characterized the EASY-spray containing a $50 \mathrm{~cm}$ column containing $<2 \mu \mathrm{m}$ particles and found that the system allowed 3000 proteins to be identified in 90 minutes. We then asked the question whether a fast and sensitive online 2D SCX-RP UHPLC-MS/MS workflow could compete with 1D long gradient analyses, using total analysis time versus proteome coverage and sample usage as benchmark parameters. The 2D LC-MS strategy consisted of the EASY-spray system that had been augmented by the addition of an SCX column. The conversion was made facile since no additional valves were required and by the use of components containing viper fittings. We benchmarked the system using a human cell lysate digest $(<10 \mu \mathrm{g})$. The 2D SCX-RP UHPLC-MS/MS workflow allowed the identification of almost 37000 unique peptides and 6000 proteins in a total analysis time of $\sim 7$ hours. On the same system a 1D RP UHPLC-MS/MS workflow plateaued at only 20000 peptides and 4400 unique proteins and required approx. 8 hours of analysis time. Furthermore, the 2D workflow could continue to increase the proteome coverage with longer analysis times, in fact with a 21 hour analysis we identified 56600 unique peptides and $>7500$ proteins. We report, here, that with this fast online SCX-RP UHPLC-MS/MS workflow, the proteome coverage can be substantially extended without significantly compromising the analysis time and sample usage.

\section{Introduction}

A major goal in mass spectrometry based proteomics is the complete characterization of the proteome that is used to detect/observe all proteins in all their proteoforms. The main analytical obstacles to reach this goal can be classified into three areas: lack of sensitivity, complexity and insufficient dynamic range. At present most in-depth analyses of proteomes employ 'shotgun strategies', ${ }^{1}$ where all proteins are extracted

${ }^{a}$ Biomolecular Mass Spectrometry and Proteomics, Bijvoet Center for Biomolecular Research and Utrecht Institute for Pharmaceutical Sciences, University of Utrecht, Padualaan 8, 3584 CH Utrecht, The Netherlands. E-mail: shabaz.mohammed@ chem.ox.ac.uk;a.j.r.heck@uu.nl

${ }^{b}$ Netherlands Proteomics Centre, Padualaan 8, 3584 CH, Utrecht, The Netherlands ${ }^{c}$ Thermo Fisher Scientific, Edisonsvej 4, DK-500o Odense C, Denmark

${ }^{d}$ Chemistry Research Laboratory, Department of Chemistry, University of Oxford, Mansfield Road, OX1 3TA, Oxford, U. K.

${ }^{e}$ Department of Biochemistry, University of Oxford, South Parks Road, OX1 3QU, Oxford, $U . K$

$\dagger$ Electronic supplementary information (ESI) available. See DOI: 10.1039/c4an01568a from the cells, digested with trypsin, whereafter the resulting complex peptide mixture is subjected to a form of liquid chromatography (LC)-mass spectrometry (MS) analysis. ${ }^{2}$ These two symbiotic analytical technologies (liquid chromatography and mass spectrometry) have improved immensely in the last decade. Mass spectrometry has advanced in many aspects, i.e. new peptide sequencing techniques, sequencing speed, mass resolution, sensitivity and dynamic range in ion detection. ${ }^{3-12}$ The advances in MS, particularly the speed, have meant that the overall performance of a proteomic LC-MS analysis would progress with increased resolution power of the upstream separation approach. Furthermore, higher resolving power would increase the chance of detecting low-abundant peptides, including those that are very similar in the mass to charge $(\mathrm{m} / \mathrm{z})$ ratio. In single dimensional (1D) LC-MS experiments, for which normally C18 materials are used, longer columns with smaller internal diameters (ID) and particle sizes have been introduced to increase the resolving power, even though such modifications lead to a dramatic increase of the back pressure on the system. ${ }^{13}$ Jorgenson et al. pioneered ultra-high pressure liquid chromatography (UHPLC) using long columns with smaller ID 
and sub-2 $\mu$ m particles. ${ }^{\mathbf{1 4 , 1 5}}$ Subsequently, Smith and co-workers provided compelling evidence of the utility of UHPLC for the proteomic community. ${ }^{\mathbf{1 6}}$ Nowadays, UHPLC systems which can hold up pressures of about 1000 bar have become commercially available and have been implemented in proteomic workflows by an increasing number of laboratories. Illustratively, Köcher et al. showed that a peak capacity of almost 700 (with a 10 hour gradient) can be achieved through the use of $50 \mathrm{~cm}$ columns and $2 \mu \mathrm{m}$ particles. ${ }^{17}$ One-dimensional RP-based UHPLC combined with the latest MS/MS technologies have extended the achievable depth in proteome coverage. Several groups have demonstrated that a few thousand proteins can now be detected in a single LC-MS/MS run using UHPLC with long columns, <2 micron particles and extended gradients. ${ }^{18-22}$

There is a very direct link between the proteome depth and the peak capacity of the chromatographic system. ${ }^{17}$ This relationship highlights the need for even higher peak capacities since full proteome analyses (in terms of the number of proteins and peptides) are still not being achieved. Although the current 1D LC-MS/MS workflows are powerful, it is still evident that no single dimensional approach has the appropriate requirements to achieve this ultimate goal. Offline multidimensional approaches, ideally using orthogonal separation principles, ${ }^{23,24}$ provide the ideal way of dramatically increasing the overall peak capacity, but typically that comes at the expense of sample loss and total MS analysis time. ${ }^{25}$

The pioneering work of Yates and co-workers ${ }^{26}$ whereby they coupled SCX to RP LC-MS/MS analysis ('MudPIT'), both in offline and online workflows, became the template for many $2 \mathrm{D}$ LC-MS/MS approaches. In MudPIT, the peptides bind first onto the SCX material and are eluted to the RP through several steps of solvent injections containing increasing concentrations of volatile salt. In between the salt steps a gradient of ACN is applied for the separation of the peptides that became bound to the RP material. ${ }^{26}$ The original MudPIT approach has been further developed, for instance, by introducing a triphasic version of the capillary containing RP-SCX-RP particles or using mixed phase ion exchange columns. ${ }^{27,28}$ Here, the first RP operates as a trap, thus avoiding the sample loss during the needed offline desalting step prior to LC-MS/MS analysis. ${ }^{29}$ Yates and co-workers also demonstrated a MudPIT system operating in the UHPLC regime ${ }^{30}$ several years before UHPLC systems were commercialized.

Despite the excellent performance of MudPIT, offline systems are still more popular because they are perceived as less difficult to build and control. In valve switching approaches, the two columns are not directly connected, allowing higher flexibility in the choice of the solvents. Additionally, first dimension SCX columns with an ID bigger than the RP second dimension column can be employed, so that a higher (and more appropriate) amount of sample can be loaded. ${ }^{25,31}$ Often, both in MudPIT and column switching approaches, a RP-based trap column is placed in between the SCX and RP columns used for the peptide separation. This allows to concentrate and desalt the peptides eluted from the SCX column, adding extra flexibility and giving the possibility to use solvents containing either volatile ${ }^{32}$ or non-volatile salts. ${ }^{33}$ Gebler et al. $^{34}$ and more recently, Kislinger et $a l .{ }^{35}$ demonstrated that commercial systems can be modified without too much effort into a 2D LC system where salt plugs were injected directly from the auto-sampler. In this way, the setup could be simplified because the solvent containing the salt does not need to be delivered by additional pumps.

Here, we demonstrate that the EASY-spray design can easily be augmented into a 2D LC configuration. We extend the platform by introducing an SCX trap (with very few new connections) and by using nanoViper fittings. Our design requires no extra pumps for the release of the SCX solvents, and can be switched rapidly from $1 \mathrm{D}$ to $2 \mathrm{D}$ and vice versa. We also show that this fast online 2D SCX-RP UHPLC-MS/MS workflow remarkably extends the proteome coverage when compared to $1 \mathrm{D}$ approaches, notably without compromising the analysis time and sample usage.

\section{Experimental section}

\section{Materials}

The complete Mini EDTA-free protease inhibitor cocktail and phosStop phosphatase inhibitor cocktail were obtained from Roche Diagnostic (Mannheim, DE), lysyl endopeptidase (Lys-C) from Wako (Richmond, VA, USA) and trypsin endopeptidase from Promega (Madison, WI, USA). Iodoacetamide, DL-dithiothreitol (DTT), ammonium bicarbonate (AMBIC) and ammonium acetate were purchased from Sigma Aldrich (Steinheim, DE). Urea, formic acid (FA) and dimethylsulfoxyde (DMSO) were obtained from Merck (Darmstadt, DE). HPLC grade acetonitrile (ACN) was obtained from Biosolve BV (Valkenswaard, NL). Bradford protein assay was purchased from BioRad Laboratories (Hercules, CA) and high purity water was obtained from a Millipore Milli-Q system (Billerica, MA).

\section{Preparation of the HEK293 digest}

HEK293 cells were re-suspended in lysis buffer composed of $8 \mathrm{M}$ urea, $50 \mathrm{mM}$ AMBIC pH 8, 1 tablet of PhosStop phosphatase inhibitors, and 1 tablet of the complete Mini EDTA-free protease inhibitor cocktail. The cell lysate was sonicated 3 times on ice. After, centrifugation at $20000 \mathrm{~g}$ at $4{ }^{\circ} \mathrm{C}$ for 20 minutes the soluble protein fraction was separated from the insoluble protein fraction. The soluble fraction was collected and the protein concentration of the lysate was determined by Bradford protein assay. Proteins were reduced with $2 \mathrm{mM}$ DTT at $56^{\circ} \mathrm{C}$ for 25 minutes, followed by alkylation with iodoacetamide $(4 \mathrm{mM})$ at room temperature for 30 minutes in the dark. After the alkylation step digestion was carried out with a first step of Lys$\mathrm{C}$ for 4 hours at $37{ }^{\circ} \mathrm{C}$ with a protein to enzyme ratio of $75: 1$ $(\mathrm{w} / \mathrm{w})$. Subsequently, the sample was diluted 4 times with $50 \mathrm{mM}$ AMBIC to a urea concentration of $2 \mathrm{M}$. The second step of digestion was performed with trypsin overnight at $37^{\circ} \mathrm{C}$ with a substrate to enzyme ratio of $100: 1(\mathrm{w} / \mathrm{w})$. After digestion the sample was acidified with $10 \%$ FA.

\section{D LC setup and experiments}

LC separation was performed with an EASY-spray system (Thermo Scientific, Odense, DK) consisting of a $50 \mathrm{~cm}, 75 \mu \mathrm{m}$ ID 
PepMap RSLC, C18, $100 \AA$ A, $2 \mu \mathrm{m}$ particles which was connected to an Easy-nLC Ultra UHPLC system (Thermo Scientific, Odense, DK).

The spray emitter was set at $1.9 \mathrm{kV}$ and the column was heated to $30{ }^{\circ} \mathrm{C}$. The sample was picked up from the autosampler vial plate and loaded into the loop (Fig. 1A) using the Pump S at $20 \mu \mathrm{l} \mathrm{min}^{-1}$ with solvent A (99.9\% water, $0.1 \% \mathrm{FA}$ ). During the sample pick up, $5 \mu \mathrm{l}$ and $1 \mu \mathrm{l}$ of solvent A were respectively used to equilibrate the pre-column and the analytical column at the controlled back pressure of 700 bar. The sample was loaded (Fig. 1B) from the loop on the backflushed trap column (Thermo, PepMap RSLC, C18, $100 \AA$ A, $5 \mu \mathrm{m}$ particles packed in $5 \mathrm{~mm}$ trap column with $300 \mu \mathrm{m}$ ID) and the trap was connected to waste for the time needed to wash with 20 $\mu \mathrm{l}$ of solvent A. The pre-column, analytical column equilibration and loading were back pressure controlled, and therefore the time employed to perform these steps can be prone to little variance. After the loading/washing step the back flushed trap column was then switched online with the analytical column (Fig. 1C) and a gradient of solvents A and B (99.9\% ACN, 0.1\% FA) was started.

The gradient for separation ranged from 7 to $30 \%$ of solvent B respectively in $22,37,67,157,214,334,454$ and 574 minutes

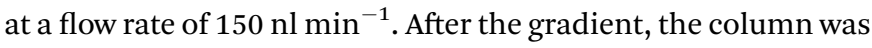
washed for 2 minutes by increasing the buffer B concentration to $100 \%$ followed by conditioning the system with $93 \%$ buffer A for at least 15 minutes. All the steps led to an analysis time of $\sim 45, \sim 60, \sim 90, \sim 180, \sim 240, \sim 360, \sim 480$ and $\sim 600 \mathrm{~min}$. The total analysis time includes washing steps, pre-column equilibration, analytical column equilibration and loading, which in each run approximately took 20-25 $\mathrm{min}$.

With analysis times of 45, 60, 90 and 180 minute lengths, $1 \mu \mathrm{g}$ of protein digest was injected while for the longer runs of 240, 360, 480 and 600 minutes, $4 \mu \mathrm{g}$ were injected in order to have a similar total ion current in every run.

\section{D LC setup and automated 6 salt plugs experiments}

The 2D experiments were performed using the same UHPLC system, trap and analytical column as described for the 1D experiments (Fig. 1). In order to implement a 2D setup we added a SCX trap column (Luna, $5 \mu \mathrm{m}, 100 \AA$, $100 \mu \mathrm{m}$ ID, $5 \mathrm{~cm}$, Phenomenex, Utrecht, NL) (dashed box in Fig. 1) in between the sample line and the $\mathrm{W}$ valve (Fig. 1). (An SCX material with similar performance to the one used in this work is also commercially available and can be purchased as a readymade column from Thermo Scientific.) In all experiments $10 \mu \mathrm{g}$ of HEK293 digest was used. The sample was transferred from the auto-sampler to the sample loop (Fig. 1A) with solvent $\mathrm{S}(0.05 \%$ FA and 5\% DMSO). The sample was loaded onto the SCX trap

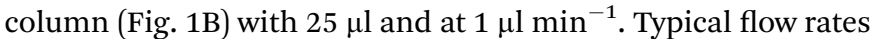
are an order of magnitude higher for loading but we found that decreasing the flow rate for SCX trapping helped to quantitatively trap the peptides on the SCX material. The peptides that did not bind the SCX trap column (flow through) were trapped on the back-flushed reversed phase trap column. Switching the W valve switch connects the trap column with the analytical

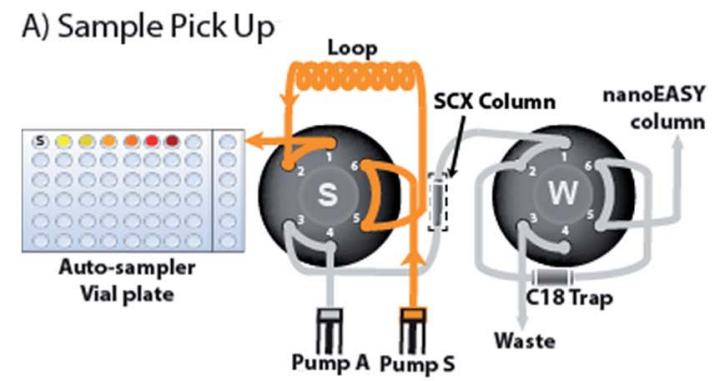

B) Loading Sample Loop
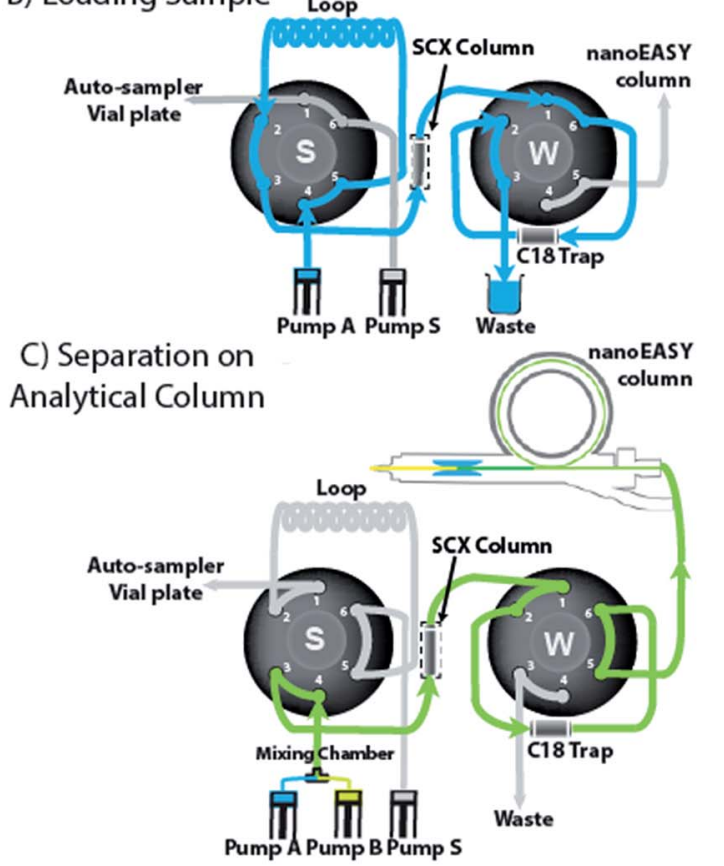

Fig. 1 Description of the 1D and 2D EASY-spray configurations. An SCX trap column (dashed box) between valves $S$ and $W$ is the difference between the 1D and 2D setups. (A) Sample pick up: the sample or the salt plugs are picked up from the auto-sampler vial plate and loaded on the loop using pump S. (B) The sample is carried from the loop to the C18 trap in the 1D fashion and from the loop to the SCX column for the $2 \mathrm{D}$ strategy. In the $2 \mathrm{D}$ setup the salt plugs are subsequently injected, populations of peptides with increasing net charges are displaced from the SCX column and bind the C18 trap. (C) The peptides are then eluted from the back-flushed C18 trap column and separated on the nanoEASY-spray column with a gradient of $A$ and $B$ solvents.

column and a gradient of solvents A and B, as described above for the 1D experiments, was applied for separation (Fig. 1C). We used 37 min gradients for short 2D experiments and $157 \mathrm{~min}$ gradients for the long 2D experiments. The above described steps were repeated for each of the injected salt steps. The six salt plugs contained ammonium acetate at concentrations of 5 $\mathrm{mM}, 10 \mathrm{mM}, 20 \mathrm{mM}, 50 \mathrm{mM}, 100 \mathrm{mM}, 500 \mathrm{mM}, 5 \%$ of $\mathrm{ACN}^{36}$ and $0.1 \%$ of FA. Each injection contained $18 \mu \mathrm{l}$ of salt solution (Fig. 1A) which was transferred onto the SCX trap column. The total volume of sample loading (Fig. 1B) was $40 \mu \mathrm{l}$ (i.e. $18 \mu \mathrm{l}$ of salt and $22 \mu \mathrm{l}$ of solvent A) to ensure extensive washing. The total analysis time for the $2 \mathrm{D}$ short experiment amounted 
approx. to $\sim 420 \mathrm{~min}$ while for the long $2 \mathrm{D}$ experiment to $\sim 1260 \mathrm{~min}$.

\section{Mass spectrometric conditions}

Mass spectra were acquired with an Orbitrap Q-Exactive mass spectrometer (Thermo Scientific, San Jose, CA) in a datadependent mode, with automatic switching between MS and MS/MS scans using a top 10 method, where the 10 most abundant precursors were chosen for fragmentation in every MS scan. MS spectra were acquired in positive mode at a resolution of 35000 with a scan range going from 350 to $1500 \mathrm{~m} / \mathrm{z}$. For the full scans the AGC target was set to $3 \times 10^{6}$ ions and the maximum injection time was set to $250 \mathrm{~ms}$. The precursor ions selected for MS2 scans were then fragmented by high-energy collision dissociation (HCD) with the energy set at 25 NCE. Ion selection was performed at $1.5 \mathrm{~m} / \mathrm{z}$ and the intensity threshold was set to $4.2 \times 10^{3}$ with a charge exclusion of $z=1$ ion. The MS/ MS spectra were acquired with a fixed first mass of $180 \mathrm{~m} / \mathrm{z}$, a resolution of 17500 , an AGC value of $5 \times 10^{4}$ ions and a maximum injection time of $120 \mathrm{~ms}$. The dynamic exclusion varied in each method due to the chromatographic performance. Increasing gradients generated wider peak widths, thus in each method the dynamic exclusion was changed accordingly to the average peak width. For the methods with 45, 60, 90, 180, 240, 360, 480 and 600 minute analysis times, the dynamic exclusion was set to $10,15,20,30,40,40,40,50$ and $50 \mathrm{~s}$, respectively.

\section{Data analysis}

Each raw data file was processed and quantified using Proteome Discoverer (version 1.3.0.339, Thermo Scientific). The Top $N$ Peaks filter was selected, where the 10 most abundant peaks in a mass window of $100 \mathrm{Da}$ alongside a signal-to-noise threshold of 1.5 were parsed. All generated peak lists were searched using Mascot software (version 2.4.1 Matrix Science). Data were searched against the UniProt-SwissProt version 2010-12 for humans. The database search was performed with the following parameters: a mass tolerance of $\pm 50 \mathrm{ppm}$ for precursor masses; \pm 0.05 Da fragment ions, allowing two missed cleavages and cysteine carbamidomethylation as fixed modifications. Methionine oxidation, $\mathrm{N}$-terminal acetylation, phosphorylation on serine, threonine and tyrosine were set as variable modifications. The scoring of phosphorylation sites of the identified phosphopeptides was performed by using the phosphoRS ${ }^{37}$ algorithm (version 2.0) implemented in Proteome Discoverer. The enzyme was specified as trypsin while the fragment ion type was specified as ESI-QUAD-TOF. Percolator ${ }^{36,38}$ calculated the target FDR with a strict cut-off of 0.01 . The identified and quantified peptides were first filtered for high confidence (FDR below 1\%) and then all the results were combined and further filtered with the following criteria: the Mascot ion score of at least 20 on peptides and proteins, maximum peptide rank 1, maximum search engine rank 1 and pRS isoform confidence probability of at least $75 \%$. The precursor ion area detection node was added for the 1D 180 and 600 min triplicates and the $2 \mathrm{D}$ experiments (1260 min and $420 \mathrm{~min}$ total analysis times), in order to obtain the area under the curve of the proteins as the sum of the three most intense peptides of a given protein. ${ }^{\mathbf{3 9 , 4 0}}$

The mass spectrometry proteomic data have been deposited to the ProteomeXchange Consortium (http://www.proteomexchange. org) via the PRIDE partner repository ${ }^{\mathbf{4 1}}$ with the dataset identifier PXD000705. Reviewer account: username: reviewer37669@ ebi.ac.uk; password: UatCrcr1. To access the data please visit: http:// tinyurl.com/od4hwsm.

\section{Results and discussion}

\section{Evaluation of the performance of the EASY-spray platform in a 1D workflow with a back-flush configuration}

The EASY-spray technology ${ }^{\mathbf{4 2}}$ consists of a plug and play source and a single piece that contains an integrated (and disposable) analytical column, a column heater and an emitter (Fig. 1). The configuration used in this work contained a $50 \mathrm{~cm}, 75 \mu \mathrm{m}$ ID analytical column (Acclaim PepMap RSLC C18, $2 \mu \mathrm{m}$ ) that was kept at $30{ }^{\circ} \mathrm{C}$. In order to decouple SCX from the RP analytical column on the commercial EASY-LC system, and therefore increase the flexibility of our $2 \mathrm{D}$ system, we opted to use a valve switching configuration requiring the RP trap column to operate in back-flush mode (Fig. 1), which is already a well-established configuration in proteomic workflows. ${ }^{43-45}$ We took a human cell lysate digest to benchmark the performance and assessed variable gradient times ranging from 22 to $574 \mathrm{~min}$ (see Materials and methods). All these 1D analyses were performed in triplicate. The results as given by the number of unique peptides and proteins detected are shown in Fig. 2 and Table S1. $\dagger$ For instance, using a 157 minute gradient $(\sim 180 \mathrm{~min}$

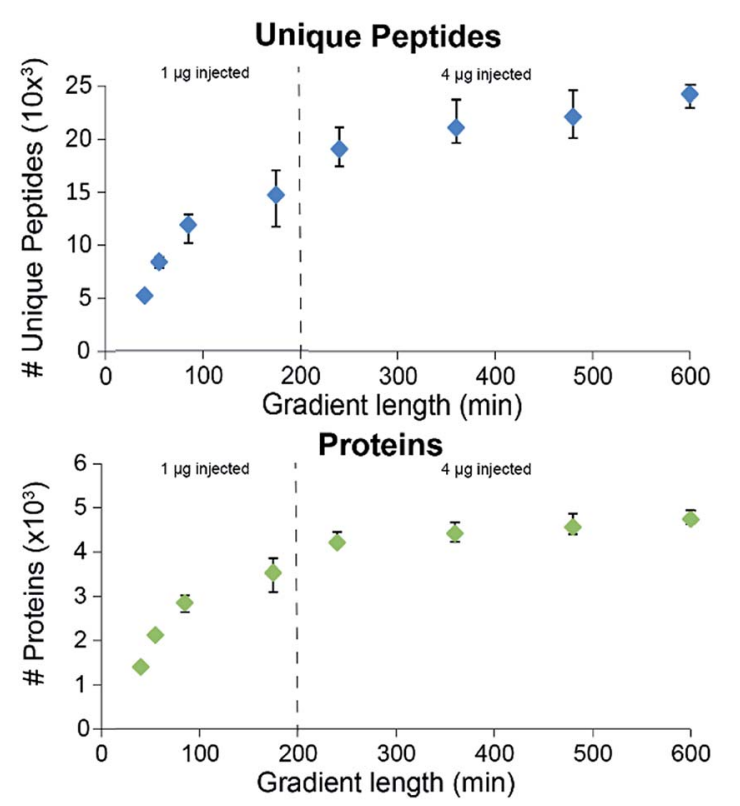

Fig. 2 Average number of peptides and proteins identified in the 1D triplicates. The top graph reports the number of unique peptides identified ( $Y$ axis), an average of three replicates, for each of the gradient lengths applied ( $X$ axis). The graph on the bottom represents the average number of unique proteins identified, from three replicates, for each applied gradient length. 
analysis time) we identified, on average, 14575 peptides per run and 3520 proteins, while extending the gradient time to $574 \mathrm{~min}$ ( $\sim 600 \mathrm{~min}$ analysis time) led to an increase in identification of 24367 peptides and 4626 proteins. The data, including the column peak capacity, follow closely the results reported by Köcher et al., ${ }^{\mathbf{4 6}}$ unsurprisingly since the column in the EASYspray platform is constructed of the same packing material, by the same manufacturer and has the same dimensions. Moreover, these results are in line with recently reported data of alternative UHPLC designs ${ }^{\mathbf{1 8 , 1 9 , 4 2 , 4 6}}$ from which we conclude that 1D back-flush configuration shows a highly competitive performance. We further tested the stochastic nature of data dependent acquisition (DDA) in MS analysis, wherein peptide fragmentation analysis is triggered on the most abundant ions in the full scans. This behavior is evident in our data as, when combining the triplicate analysis of the $\sim 180$ min runs (i.e. $\sim 540$ min total analysis time), we increased our coverage to a total of 24367 peptides and 4369 proteins. Similarly 37805 peptides, and 5646 proteins could be detected combining the data of three $600 \mathrm{~min}$ runs (i.e. $1800 \mathrm{~min}$ total analysis time) (Table S2 $\dagger$ ).

As also shown by $\mathrm{us}^{18}$ and others ${ }^{12,18,20,46}$ and visualized by our data in Fig. 2, in a 1D LC-MS/MS approach using ultra-long gradients the amount of detected "new" unique peptides and proteins levels off rapidly, certainly beyond $214 \mathrm{~min}$ of the gradient time in our system. These data indicate that other dimensions of separation are needed to increase the proteome coverage.

\section{Evaluation of the EASY-spray platform in a 2D SCX-RP workflow}

Our aim was to design an automated online multidimensional UHPLC workflow, requiring minimal facile changes, having competitive performance in parameters such as the proteome depth, total analyses time and sensitivity. In our design no extra pumps are needed to supply the salt solution as the plugs are injected directly from the auto-sampler (Fig. 1A). In this system, switching from the 1D to 2D setup is straightforward, as just an SCX trap column is connected via a nanoViper fitting using a zero dead volume connector (dashed box, Fig. 1). After an initial evaluation we found that the optimal salt plug concentrations and compositions were consistent with previous MudPIT experiments, ${ }^{26,30,35}$ unsurprisingly since we were utilizing an SCX column of similar composition and dimensions. To further evaluate the system we used aliquots $(10 \mu \mathrm{g})$ of the same HEK293 digest. The 2D configuration was tested with two types of analyses; one set were aimed at evaluating the performance when employing short (37 min) second dimension RP gradients, while the second set of experiments used longer RP gradients (157 min). Total analysis times for the two 2D SCX-RP experiments were $\sim 420$ and $\sim 1260$ min, respectively.

The MS analyses of the flow through revealed relatively few regular unmodified tryptic peptides indicating good retention by the SCX material (Fig. 3). Moreover, a more careful observation of the peptides eluted in the flow through and $5 \mathrm{mM}$ salt plug showed that the majority contained either an acetyl group on the N-terminus or a phosphate group (Fig. S1†), in agreement with previous observations by Alpert et al. ${ }^{47}$ Salt plugs of 10, 20 and $50 \mathrm{mM}$ ammonium acetate produced highly complex
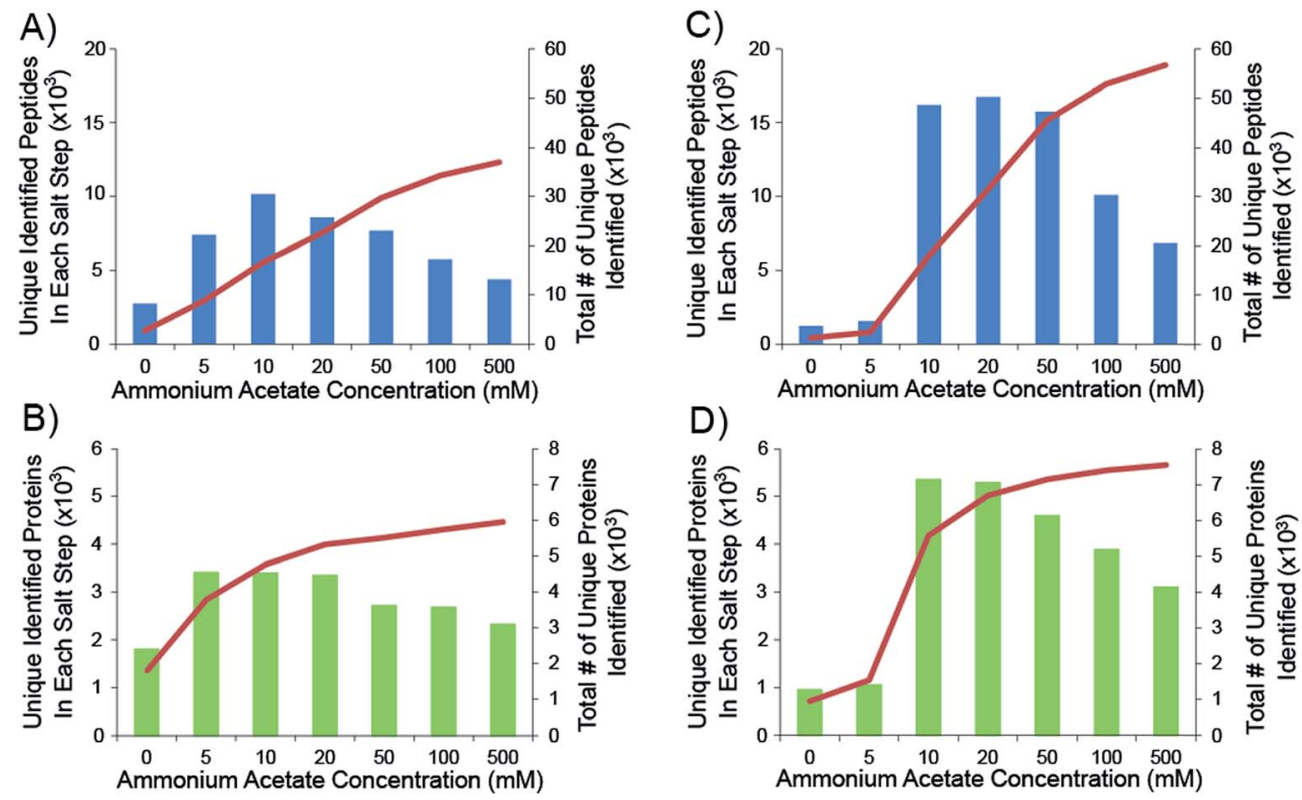

Fig. 3 Total and per fraction number of unique peptides and proteins identified in the 2D experiments. (A) The plot shows for the 2D short experiment ( $420 \mathrm{~min}$ of analysis time) the number of unique peptides identified for each salt plug injected ( $0 \mathrm{mM}$ to $500 \mathrm{mM})$ and with the red line the cumulative number of unique peptides identified. (B) The number of unique proteins for each salt plug and the cumulative number of unique proteins identified is presented for the 2D short experiment. (C) The number of unique peptides for each salt plug and the cumulative number of unique peptides identified is shown for the 2D long experiment (1260 min analysis time). (D) The number of unique proteins for each salt plug and the cumulative number of unique proteins identified is shown for the 2D long experiment. 
peptide mixtures, causing identification rates to be essentially controlled by the length of the reversed phase gradient. The number of unique peptides and proteins identified per fraction, and cumulatively, are summarized in Fig. 3. Each subsequent salt plug produced diminishing returns. Pleasingly, we observed that the peak capacity of the RP column (above 400 for 157 min gradients using peak widths at $4 \sigma$ or at $13.4 \%$ peak height) remained intact after the injection of salt plugs and typical for this material and column dimensions. ${ }^{17}$ Peptides identified in multiple fractions, numbered no more than $20 \%$ of any fraction, suggesting a mild issue with carryover or poor fractionation. Based on these data, we attempted a third experiment where we chose a gradient length that is linked to the complexity of the salt plug. We chose a 37 min gradient for the 0 and $5 \mathrm{mM}$ fractions, $214 \mathrm{~min}$ for the 10, 20, 50 and 100 $\mathrm{mM}$ fractions and $157 \mathrm{~min}$ for the $500 \mathrm{mM}$ salt plug, for a total analysis time of $\sim 1260 \mathrm{~min}$. Unfortunately, the optimal gradient for each fraction had a negligible effect on the end result (Fig. S2†).

In the short 2D experiment we identified 36943 unique peptides and 5958 proteins while with the long $2 \mathrm{D}$ experiment we identified 56600 unique peptides and 7565 proteins (Fig. 3 and Table S $3 \dagger$ ). These results are not only favorable in the total analysis time compared to the triplicate 1D LC-MS/MS analyses ( $\sim 540$ and $\sim 1800 \mathrm{~min}$ ) described above, but also in the achieved proteome depth. Using $\sim 1260 \mathrm{~min}$ of total analysis time our 2D LC-MS/MS approach identifies 34\% more proteins than the $\sim 1800 \mathrm{~min}$ triplicate 1D LC-MS/MS approach. See Table S4† for an overview of all results in the context of the sample amount, time and configuration.

We next investigated the overlap in identified peptides comparing one-to-one the cumulative data of the three short and long analysis time triplicate $1 \mathrm{D}$ experiments, with the short and more extended $2 \mathrm{D}$ experiments, respectively (Fig. S3†). The

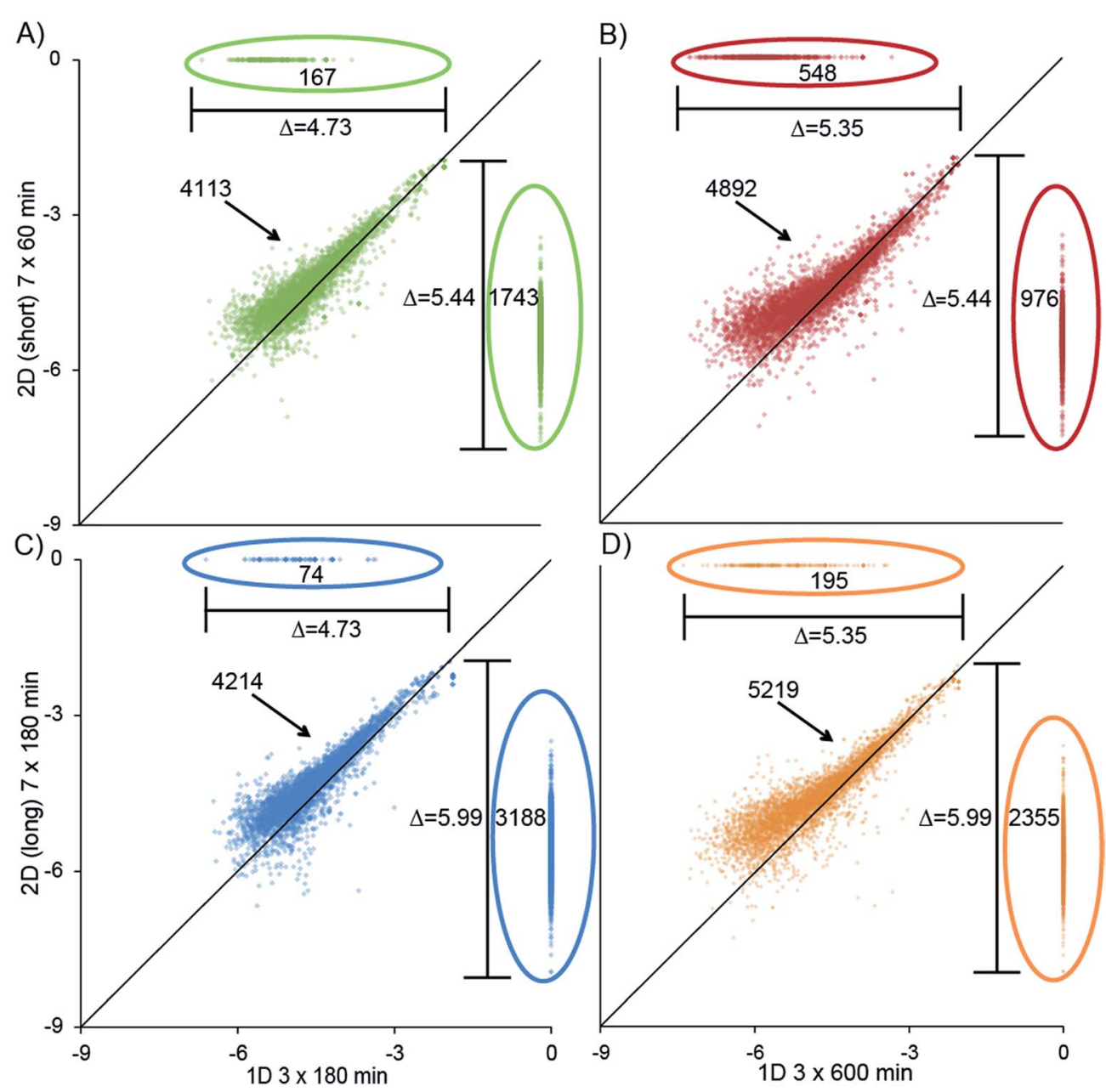

Fig. 4 Normalized protein intensities $\left(\log _{10}\right)$ for the $2 D$ and $1 D$ combined triplicate experiments. The plots show on the $Y$ axis the normalized intensity $\left(\log _{10}\right)$ of proteins quantified with the $2 \mathrm{D}$ short $(A$ and $B)$ and long $(C$ and $D)$ experiment and on the $X$ axis the normalized protein intensities on the logarithmic scale for the $1 \mathrm{D} 180$ ( $A$ and $C$ ) and $600 \mathrm{~min}$ ( $\mathrm{B}$ and $\mathrm{D}$ ) combined triplicates. The clouds in the middle of the graphs are the overlapping quantified proteins among the $2 \mathrm{D}$ and $1 \mathrm{D}$ experiments. On the $X$ axis (top part) the graphs show the number of proteins uniquely quantified by the 1D $180 \mathrm{~min}(\mathrm{~A}$ and $\mathrm{C}$ ) and $600 \mathrm{~min}$ ( $\mathrm{B}$ and $\mathrm{D}$ ) combined triplicates while on the $Y$ axis (right side) the number of proteins only quantified with the $2 \mathrm{D}$ short $(A$ and $B)$ and long $(C$ and $D)$ experiment are shown. The horizontal bars illustrate the dynamic range of the $1 D$ $180 \mathrm{~min}(\mathrm{~A}$ and $\mathrm{C}$ ) and $600 \mathrm{~min}(\mathrm{~B}$ and $\mathrm{D})$ triplicate experiments while the vertical the dynamic ranges for the 2D experiments. 
choice of 1D runs was based on the analysis time. We not only identified, in the extended 2D LC experiment, the majority of proteins and peptides found in the two 1D experiments, but we also significantly increased the number of peptides and proteins identified (Fig. S3(C), (D), (G) and (H)†). This performance of the longer gradient time 2D experiment is likely due to the combined effect of the fractionation and the longer RP gradient used i.e. a larger effective peak capacity. It is worthy to note that even the short version 2D experiment could rival with the long-gradient 1D combined triplicates (Fig. S3(A), (B), (E) and $(\mathrm{F}) \dagger)$.

To further demonstrate the advantageous effect of the online multidimensional UHPLC separation we then investigated the protein abundances as reflected by the ion currents of their top3 peptides. ${ }^{39,40}$ In Fig. 4 the intensities of the proteins are plotted as extracted from the data out of the $2 \mathrm{D}$ and $1 \mathrm{D}$ experiments. The dots at the top represent proteins only observed in the 1D experiment while the dots at the right side of the plots represent proteins uniquely identified in the $2 \mathrm{D}$ experiment. Pleasingly, in general the data points follow a straight line, indicating that the derived protein abundance is in agreement among the different experiments. However, it is clear from these data that high abundant proteins have typical ratios close to $1: 1$, while most of the medium to low abundant proteins have higher estimated abundance in the 2D experiments, making them appear above the straight line. This observation is likely the results of the higher resolution obtained in the $2 \mathrm{D}$ approach, which decreases ion suppression elevating ${ }^{48,49}$ ion currents for peptides coeluting with high abundant ones in the 1D workflow. Fig. 4 also shows the dynamic range of the different experiments calculated as the difference in the intensity between the highest abundant protein and the lowest. As expected the highest dynamic range belonged to the long $2 \mathrm{D}$ experiment followed by the short 2D one. The 1D 600 min triplicate showed a similar dynamic range to the short $2 \mathrm{D}$ experiment despite its much longer analysis time, while 1D $180 \mathrm{~min}$ triplicate performed poorest. It seems that a single $2 \mathrm{D}$ experiment of a similar analytical time outperforms a set of $1 \mathrm{D}$ experiments. Moreover, the mass spectrometer itself is a key component which heavily influences overall proteome performance. Recent improvements in mass spectrometers such as those found in the Orbitrap Fusion ${ }^{50}$ will also have a major influence. We envisage that these mass spectrometers will equally benefit (in terms of proteome coverage boost) when the peak capacity of the separation is increased by switching from $1 \mathrm{D}$ to $2 \mathrm{D}$ LC setups.

\section{Conclusion}

Here, we demonstrate that the EASY-spray system is a powerful and versatile platform. We report on a plug and play online multidimensional SCX-RP UHPLC system that does not require additional pumps for the elution of the salt plugs and is capable of identifying over 7500 proteins when using a Q-Exactive. A major benefit of our setup is that minimal changes are required to modify the 1D LC-MS system. Therefore, we believe this system can be easily constructed by any proteomic laboratory.

\section{Acknowledgements}

The authors acknowledge all members of the Heck-group. This research was performed within the framework of PRIME-XS, grant number 262067, funded by the European Union 7th Framework Program. Additionally, the Netherlands Organization for Scientific Research (NWO) supported large scale proteomics facility Proteins@Work (project 184.032.201) embedded in the Netherlands Proteomics Centre is kindly acknowledged for financial support as well as the Netherlands Organization for Scientific Research (NWO) with the VIDI grant for SM (700.10.429).

\section{References}

$1 \mathrm{~J}$. R. Yates, Mass spectrometry and the age of the proteome, $J$. Mass Spectrom., 1998, 33(1), 1-19.

2 Y. Zhang, B. R. Fonslow, B. Shan, M. C. Baek and J. R. Yates, Protein analysis by shotgun/bottom-up proteomics, Chem. Rev., 2013, 113(4), 2343-2394.

3 R. A. Zubarev and A. Makarov, Orbitrap mass spectrometry, Anal. Chem., 2013, 85(11), 5288-5296.

4 A. Michalski, E. Damoc, J. P. Hauschild, O. Lange, A. Wieghaus, A. Makarov, N. Nagaraj, J. Cox, M. Mann and S. Horning, Mass spectrometry-based proteomics using Q Exactive, a high-performance benchtop quadrupole Orbitrap mass spectrometer, Mol. Cell. Proteomics, 2011, 10(9), DOI: 10.1074/mcp.M111.011015.

5 A. Makarov, E. Denisov, O. Lange and S. Horning, Dynamic range of mass accuracy in LTQ Orbitrap hybrid mass spectrometer, J. Am. Soc. Mass Spectrom., 2006, 17(7), 977982.

6 J. V. Olsen, J. C. Schwartz, J. Griep-Raming, M. L. Nielsen, E. Damoc, E. Denisov, O. Lange, P. Remes, D. Taylor, M. Splendore, E. R. Wouters, M. Senko, A. Makarov, M. Mann and S. Horning, A dual pressure linear ion trap Orbitrap instrument with very high sequencing speed, Mol. Cell. Proteomics, 2009, 8(12), 2759-2769.

7 A. F. Altelaar, J. Munoz and A. J. Heck, Next-generation proteomics: towards an integrative view of proteome dynamics, Nat. Rev. Genet., 2013, 14(1), 35-48.

$8 \mathrm{~J}$. V. Olsen and M. Mann, Status of large-scale analysis of post-translational modifications by mass spectrometry, Mol. Cell. Proteomics, 2013, 12(12), 3444-3452.

9 G. L. Andrews, B. L. Simons, J. B. Young, A. M. Hawkridge and D. C. Muddiman, Performance characteristics of a new hybrid quadrupole time-of-flight tandem mass spectrometer (TripleTOF 5600), Anal. Chem., 2011, 83(13), 5442-5446.

10 J. E. Syka, J. J. Coon, M. J. Schroeder, J. Shabanowitz and D. F. Hunt, Peptide and protein sequence analysis by electron transfer dissociation mass spectrometry, Proc. Natl. Acad. Sci. U. S. A., 2004, 101(26), 9528-9533.

11 M. Stoeckli, P. Chaurand, D. E. Hallahan and R. M. Caprioli, Imaging mass spectrometry: a new technology for the analysis of protein expression in mammalian tissues, Nat. Med., 2001, 7(4), 493-496. 
12 T. P. Second, J. D. Blethrow, J. C. Schwartz, G. E. Merrihew, M. J. MacCoss, D. L. Swaney, J. D. Russell, J. J. Coon and V. Zabrouskov, Dual-pressure linear ion trap mass spectrometer improving the analysis of complex protein mixtures, Anal. Chem., 2009, 81(18), 7757-7765.

$13 \mathrm{~J}$. W. Jorgenson, Capillary liquid chromatography at ultrahigh pressures, Annu. Rev. Anal. Chem., 2010, 3, 129150.

14 J. E. MacNair, K. C. Lewis and J. W. Jorgenson, Ultrahighpressure reversed-phase liquid chromatography in packed capillary columns, Anal. Chem., 1997, 69(6), 983-989.

15 J. E. MacNair, K. D. Patel and J. W. Jorgenson, Ultrahighpressure reversed-phase capillary liquid chromatography: isocratic and gradient elution using columns packed with 1.0-micron particles, Anal. Chem., 1999, 71(3), 700-708.

16 Y. Shen, R. Zhao, S. J. Berger, G. A. Anderson, N. Rodriguez and R. D. Smith, High-efficiency nanoscale liquid chromatography coupled on-line with mass spectrometry using nanoelectrospray ionization for proteomics, Anal. Chem., 2002, 74(16), 4235-4249.

17 T. Köcher, R. Swart and K. Mechtler, Ultra-high-pressure RPLC hyphenated to an LTQ-Orbitrap Velos reveals a linear relation between peak capacity and number of identified peptides, Anal. Chem., 2011, 83(7), 2699-2704.

18 A. Cristobal, M. L. Hennrich, P. Giansanti, S. S. Goerdayal, A. J. Heck and S. Mohammed, In-house construction of a UHPLC system enabling the identification of over 4000 protein groups in a single analysis, Analyst, 2012, 137(15), 3541-3548.

19 S. S. Thakur, T. Geiger, B. Chatterjee, P. Bandilla, F. Fröhlich, J. Cox and M. Mann, Deep and highly sensitive proteome coverage by LC-MS/MS without prefractionation, Mol. Cell. Proteomics, 2011, 10(8), DOI: 10.1074/mcp.M110.003699.

20 F. Zhou, Y. Lu, S. B. Ficarro, J. T. Webber and J. A. Marto, Nanoflow low pressure high peak capacity single dimension LC-MS/MS platform for high-throughput, indepth analysis of mammalian proteomes, Anal. Chem., 2012, 84(11), 5133-5139.

21 R. Yamana, M. Iwasaki, M. Wakabayashi, M. Nakagawa, S. Yamanaka and Y. Ishihama, Rapid and deep profiling of human induced pluripotent stem cell proteome by oneshot NanoLC-MS/MS analysis with meter-scale monolithic silica columns, J. Proteome Res., 2013, 12(1), 214-221.

22 M. Iwasaki, N. Sugiyama, N. Tanaka and Y. Ishihama, Human proteome analysis by using reversed phase monolithic silica capillary columns with enhanced sensitivity, J. Chromatogr. A, 2012, 1228, 292-297.

23 M. Gilar, P. Olivova, A. E. Daly and J. C. Gebler, Orthogonality of separation in two-dimensional liquid chromatography, Anal. Chem., 2005, 77(19), 6426-6434.

24 M. Gilar, J. Fridrich, M. R. Schure and A. Jaworski, Comparison of orthogonality estimation methods for the two-dimensional separations of peptides, Anal. Chem., 2012, 84(20), 8722-8732.

25 S. Di Palma, M. L. Hennrich, A. J. Heck and S. Mohammed, Recent advances in peptide separation by multidimensional liquid chromatography for proteome analysis, J. Proteomics, 2012, 75(13), 3791-3813.

26 M. P. Washburn, D. Wolters and J. R. Yates, Large-scale analysis of the yeast proteome by multidimensional protein identification technology, Nat. Biotechnol., 2001, 19(3), 242-247.

27 G. P. Mommen, H. D. Meiring, A. J. Heck and A. P. de Jong, Mixed-bed ion exchange chromatography employing a saltfree $\mathrm{pH}$ gradient for improved sensitivity and compatibility in MudPIT, Anal. Chem., 2013, 85(14), 6608-6616.

28 A. Motoyama, T. Xu, C. I. Ruse, J. A. Wohlschlegel and J. R. Yates, Anion and cation mixed-bed ion exchange for enhanced multidimensional separations of peptides and phosphopeptides, Anal. Chem., 2007, 79(10), 3623-3634.

29 W. McDonald, R. Ohi, D. Miyamoto, T. Mitchison and J. Yates, Comparison of three directly coupled HPLC MS/ MS strategies for identification of proteins from complex mixtures: single-dimension LC-MS/MS, 2-phase MudPIT, and 3-phase MudPIT, Int. J. Mass Spectrom., 2002, 219(1), 245-251.

30 A. Motoyama, J. D. Venable, C. I. Ruse and J. R. Yates, Automated ultra-high-pressure multidimensional protein identification technology (UHP-MudPIT) for improved peptide identification of proteomic samples, Anal. Chem., 2006, 78(14), 5109-5118.

31 M. L. Fournier, J. M. Gilmore, S. A. Martin-Brown and M. P. Washburn, Multidimensional separations-based shotgun proteomics, Chem. Rev., 2007, 107(8), 3654-3686.

32 G. Mitulović, C. Stingl, M. Smoluch, R. Swart, J. P. Chervet, I. Steinmacher, C. Gerner and K. Mechtler, Automated, online two-dimensional nano-liquid chromatography tandem mass spectrometry for rapid analysis of complex protein digests, Proteomics, 2004, 4(9), 2545-2557.

33 E. Nägele, M. Vollmer and P. Hörth, Improved 2D nano-LC/ MS for proteomics applications: a comparative analysis using yeast proteome, J. Biomol. Tech., 2004, 15(2), 134-143.

34 H. Liu, J. W. Finch, J. A. Luongo, G. Z. Li and J. C. Gebler, Development of an online two-dimensional nano-scale liquid chromatography/mass spectrometry method for improved chromatographic performance and hydrophobic peptide recovery, J. Chromatogr. A, 2006, 1135(1), 43-51.

35 P. Taylor, P. A. Nielsen, M. B. Trelle, O. B. Hørning, M. B. Andersen, O. Vorm, M. F. Moran and T. Kislinger, Automated 2D peptide separation on a 1D nano-LC-MS system, J. Proteome Res., 2009, 8(3), 1610-1616.

36 T. Burke, C. Mant, J. Black and R. Hodges, Strong CationExchange High-Performance Liquid-Chromatography of Peptides - Effect Of Non-Specific Hydrophobic Interactions And Linearization of Peptide Retention Behavior, $J$. Chromatogr., 1989, 476, 377-389.

37 T. Taus, T. Köcher, P. Pichler, C. Paschke, A. Schmidt, C. Henrich and K. Mechtler, Universal and confident phosphorylation site localization using phosphoRS, J. Proteome Res., 2011, 10(12), 5354-5362.

38 L. Käll, J. D. Canterbury, J. Weston, W. S. Noble and M. J. MacCoss, Semi-supervised learning for peptide 
identification from shotgun proteomics datasets, Nat. Methods, 2007, 4(11), 923-925.

39 J. Grossmann, B. Roschitzki, C. Panse, C. Fortes, S. BarkowOesterreicher, D. Rutishauser and R. Schlapbach, Implementation and evaluation of relative and absolute quantification in shotgun proteomics with label-free methods, J. Proteomics, 2010, 73(9), 1740-1746.

40 J. C. Silva, M. V. Gorenstein, G. Z. Li, J. P. Vissers and S. J. Geromanos, Absolute quantification of proteins by LCMSE: a virtue of parallel MS acquisition, Mol. Cell. Proteomics, 2006, 5(1), 144-156.

41 J. A. Vizcaíno, R. G. Côté, A. Csordas, J. A. Dianes, A. Fabregat, J. M. Foster, J. Griss, E. Alpi, M. Birim, J. Contell, G. O'Kelly, A. Schoenegger, D. Ovelleiro, Y. Pérez-Riverol, F. Reisinger, D. Ríos, R. Wang and H. Hermjakob, The PRoteomics IDEntifications (PRIDE) database and associated tools: status in 2013, Nucleic Acids Res., 2013, 41(Database issue), D1063-D1069.

42 M. Pirmoradian, H. Budamgunta, K. Chingin, B. Zhang, J. Astorga-Wells and R. A. Zubarev, Rapid and deep human proteome analysis by single-dimension shotgun proteomics, Mol. Cell. Proteomics, 2013, 12(11), 3330-3338.

43 R. Giambruno, F. Grebien, A. Stukalov, C. Knoll, M. Planyavsky, E. L. Rudashevskaya, J. Colinge, G. SupertiFurga and K. L. Bennett, Affinity purification strategies for proteomic analysis of transcription factor complexes, $J$. Proteome Res., 2013, 12(9), 4018-4027.

44 I. Chamrad, U. Rix, A. Stukalov, M. Gridling, K. Parapatics, A. C. Muller, S. Altiok, J. Colinge, G. Superti-Furga, E. B. Haura and K. L. Bennett, A miniaturized chemical proteomic approach for target profiling of clinical kinase inhibitors in tumor biopsies, J. Proteome Res., 2013, 12(9), 4005-4017.

45 Y. Shen, R. J. Moore, R. Zhao, J. Blonder, D. L. Auberry, C. Masselon, L. Pasa-Tolić, K. K. Hixson, K. J. Auberry and R. D. Smith, High-efficiency on-line solid-phase extraction coupling to 15-150-microm-i.d. column liquid chromatography for proteomic analysis, Anal. Chem., 2003, 75(14), 3596-3605.

46 T. Köcher, P. Pichler, R. Swart and K. Mechtler, Analysis of protein mixtures from whole-cell extracts by single-run nanoLC-MS/MS using ultralong gradients, Nat. Protoc., 2012, 7(5), 882-890.

47 A. J. Alpert, K. Petritis, L. Kangas, R. D. Smith, K. Mechtler, G. Mitulović, S. Mohammed and A. J. Heck, Peptide orientation affects selectivity in ion-exchange chromatography, Anal. Chem., 2010, 82(12), 5253-5259.

48 R. King, R. Bonfiglio, C. Fernandez-Metzler, C. Miller-Stein and T. Olah, Mechanistic investigation of ionization suppression in electrospray ionization, J. Am. Soc. Mass Spectrom., 2000, 11(11), 942-950.

49 A. Schmidt, M. Karas and T. Dulcks, Effect of different solution flow rates on analyte ion signals in nano-ESI MS, or: when does ESI turn into nano-ESI?, J. Am. Soc. Mass Spectrom., 2003, 14(5), 492-500.

50 A. S. Hebert, A. L. Richards, D. J. Bailey, A. Ulbrich, E. E. Coughlin, M. S. Westphall and J. J. Coon, The one hour yeast proteome, Mol. Cell. Proteomics, 2014, 13(1), 339-347. 